\title{
Patient Readmissions, Emergency Visits, and Adverse Events After Software-Assisted Discharge From Hospital: Cluster Randomized Trial
}

\author{
James F. Graumlich, mD ${ }^{1,2}$ \\ Nancy L. Novotny, PhD ${ }^{1}$ \\ G. Stephen Nace, мо $^{1,2}$ \\ Himangi Kaushal, MD ${ }^{1,2}$ \\ Waleed Ibrahim-Ali, mo ${ }^{1,2}$ \\ Shoba Theivanayagam, mo ${ }^{1,2}$ \\ L. William Scheibel, $\mathrm{MD}, \mathrm{ScD}^{3}$ \\ Jean C. Aldag, PhD ${ }^{1}$
}

\author{
${ }^{1}$ Department of Medicine, University of Illinois College of Medicine, Peoria, Illinois. \\ ${ }^{2}$ Order of St. Francis (OSF)-Saint Francis Medical Center, Peoria, Illinois. \\ ${ }^{3}$ Department of Cancer Biology and Pharmacology, University of Illinois College of Medicine, Peoria, Illinois.
}

Supported by the Agency for Healthcare Research and Quality (5 R01 HS015084). Trial registration: NCT00101868 (http://clinicaltrials.gov).

Disclosure: Nothing to report.

BACKGROUND: One of the causes of postdischarge adverse events is poor discharge communication between hospital-based physicians, patients, and outpatient physicians. The value of hospital discharge software to improve communication and clinically relevant outcomes is unknown.

OBJECTIVE: To measure effects of a discharge software application of computerized physician order entry (CPOE).

DESIGN: Cluster randomized controlled trial.

SETTING: Tertiary care, teaching hospital in central Illinois.

PATIENTS: A total of 631 inpatients discharged to home with high risk for readmission.

INTERVENTION: Seventy internal medicine hospital physicians were randomly assigned (allocation concealed) to discharge software versus usual care, handwritten discharge.

MEASUREMENTS: Blinded assessment of patient readmission, emergency department visit, and postdischarge adverse event. RESULTS: A total of 590 (94\%) patients provided 6-month follow-up data. Generalized estimating equations gave intervention variable coefficients with $95 \%$ confidence interval (CI). When comparing patients assigned to discharge software versus usual care, there was no difference in hospital readmission within 6 months (37.0\% versus 37.8\%; coefficient -0.005 [95\% CI, -0.074 to 0.065 ]; $P=0.894$ ), emergency department visit within 6 months (35.4\% versus $40.6 \%$; coefficient -0.052 [95\% CI, -0.115 to 0.011$] ; P=0.108)$, or adverse event within 1 month $(7.3 \%$ versus $7.3 \%$; coefficient 0.003 [95\% CI; -0.037 to 0.043 ]; $P=0.884$ ).

CONCLUSIONS: Discharge software with CPOE did not affect readmissions, emergency department visits, or adverse events after discharge. Future studies should assess other endpoints such as patient perceptions or physician perceptions to see if discharge software has value. Journal of Hospital Medicine 2009;4:E1 1-E19. $\odot 2009$ Society of Hospital Medicine.

KEYWORDS: continuity of patient care, electronic discharge summary, health care surveys, hospital information systems, hospitalists, medical records systems-computerized, medication reconciliation, patient care transitions, patient discharge, patient satisfaction.

Adverse events occur to patients after their discharge from acute care hospitals. ${ }^{1,2}$ Most of these injuries are adverse drug events, procedure-related events, nosocomial infections, or falls. ${ }^{1}$ Postdischarge adverse events are associated with several days of symptoms, nonpermanent disability, emergency department visits, or hospital readmission. ${ }^{1,3}$ When adverse events are preventable or ameliorable, the most common root cause is poor communication between hospital personnel and either the patient or the outpatient primary care physician. ${ }^{1}$ In addition, there may be deficits in discharge processes related to assessment and communication of unresolved problems. ${ }^{1}$ Systematic reviews have shown that discharge communication is an inefficient and error-prone process. ${ }^{4-6}$
One potential solution to poor discharge communication is health information technology. ${ }^{7}$ An example of technology is discharge software with a computerized physician order entry (CPOE) system. By definition, a CPOE system is a computer-based system that automates direct entry of orders by physicians and ensures standardized, legible, and complete orders. ${ }^{8}$ The benefits of CPOE have been tested in other inpatient settings. ${ }^{8,9}$ It is logical to consider software applications with CPOE for discharge interventions. ${ }^{7}$

Several mechanisms explain the potential benefit of discharge software with CPOE. $^{7}$ Applications with $\mathrm{CPOE}$ decrease medication errors. ${ }^{8,10}$ Software with decision support could prompt physicians to enter posthospitalization 
appointment dates and orders for preventive services. ${ }^{11,12}$ Discharge software could facilitate medication reconciliation and generate patient instructions and information. ${ }^{4,13-15}$ The potential benefits of discharge software with CPOE provide a rationale for clinical trials to measure benefits.

Previous studies addressed discharge applications of health information technology. Observational studies recorded outcomes such as physician satisfaction. ${ }^{16,17}$ Prior randomized clinical trials measured quality and timeliness of discharge summaries. ${ }^{18}$ However, these previous trials did not assess clinically relevant outcomes like readmissions, emergency department visits, or adverse events. We performed a cluster-randomized trial to assess the value of a discharge software application of CPOE. The rationale for our clustered design complied with recommendations from a systematic review of discharge interventions. ${ }^{5}$ Our objective was to assess the benefit of discharge software with CPOE when used to discharge patients at high risk for repeat admission. After the intervention, we compared the rates of hospital readmission, emergency department visits, and postdischarge adverse events due to medical management.

\section{Methods}

The trial design was a cluster randomized, controlled trial with blinded outcome assessment. Follow-up occurred until 6 months after discharge from index hospitalization at a 730-bed, tertiary care, teaching hospital in central Illinois. The Peoria Institutional Review Board approved the protocol for human research.

\section{Participants}

The cluster definition was the hospital physician. Patients discharged by the physician comprised the cluster. Hospital physicians and patients were enrolled between November 2004 and January 2007. Internal medicine resident or attending physicians were eligible. We excluded hospital physicians if their assignments to inpatient duties were less than 2 months during the 27-month enrollment period. The rationale for the physician exclusion was a consequence of the patient enrollment rate of 3 to 5 patients per physician per month. Physicians with brief assignments could not achieve the goal of 9 or more patients per cluster. After physicians gave informed consent to screen their patients, trained research coordinators applied inclusion and exclusion criteria and obtained informed consent from patients. Research personnel identified all consecutive, unique, adult inpatients who were discharged to home. Patient inclusion required a probability of repeat admission (Pra) score $\geq 0.40 .{ }^{19,20}$ The Pra score came from a logistic model of age, gender, prior hospitalizations, prior doctor visits, self-rated health status, presence of informal caregiver in the home, and comorbid coronary heart disease and diabetes mellitus. Research coordinators calculated the Pra within 2 days before discharge from the index hospitalization. Other details about exclusion criteria have been published. ${ }^{21}$ If a patient's outpatient primary care physician treated the patient during the index hospitalization, then there was no perceived barrier in physician-to-physician communication and we excluded the patient.

\section{Intervention}

The research intervention was a CPOE software application that facilitated communication at the time of hospital discharge to patients, retail pharmacists, and community physicians. Details about the discharge software appeared in a previous publication. ${ }^{7}$ Software features included required fields, pick lists, standard drug doses, alerts, reminders, and online reference information. The software prompted the discharging physician to enter pending tests and order tests after discharge. Hospital physicians used the software on the day of discharge and automatically generated 4 discharge documents. The first document was a personalized letter to the outpatient physician with discharge diagnoses, reconciled medication list, diet and activity instructions, patient education materials provided, and follow-up appointments and studies. Second, the software printed legible prescriptions along with specific information for the dispensing pharmacist about changes and deletions in the patient's previous regimen. Third, the software created patient instructions with addresses and telephone numbers for follow-up appointments and tests. Fourth, the software printed a legible discharge order including all of the aforementioned information.

The control intervention was the usual care discharge process as described previously. ${ }^{7}$ Hospital physicians and ward nurses completed handwritten discharge forms on the day of discharge. The forms contained blanks for discharge diagnoses, discharge medications, medication instructions, postdischarge activities and restrictions, postdischarge diet, postdischarge diagnostic and therapeutic interventions, and appointments. Patients received handwritten copies of the forms, 1 page of which also included medication instructions and prescriptions. A previous publication gave details about the standard care available to all patients regardless of intervention. ${ }^{7}$

\section{Randomization}

The unit of randomization was the hospital physician who performed the discharge process. Random allocation was to discharge software or usual care discharge process. The randomization ratio was $1: 1$, the block size was 2 , and there was no stratification or matching. There was concealed allocation and details are available from the investigators. Hospital physicians subsequently used their randomly assigned process when discharging their patients who enrolled in the study. After random allocation, it was not possible to conceal the test or control intervention from physicians or their patients. Likewise, it was not possible to conceal the outcome ascertainment, including readmission, from the hospital physicians. 
All hospital physicians received training on the usual care discharge process. Physicians assigned to discharge software completed additional training via multimedia demonstration with 1-on-1 coaching as needed. Physicians assigned to usual care did not receive training on the discharge software and were blocked from using the software. After informed consent, patients were passive recipients of the research intervention performed by their discharging physician. Patients received the research intervention on the day of discharge from the index hospitalization.

The baseline assessment of patient characteristics occurred during the index hospitalization. Trained data abstractors recorded patient demographic data plus variables to calculate the Pra score for probability for repeat admission. We recorded additional variables because of their possible association with readmission. ${ }^{15,22-29}$ Data came from the patient or proxy for physical functioning and mental health (SF-36, Version 2; Medical Outcomes Trust, Boston, MA). Other data for predictor variables came from interviews or hospital records.

\section{Outcome Assessment}

The primary study outcome was the proportion of patients readmitted at least once within 6 months after the index hospitalization. Readmission was for any reason and included observation and full admission status. Secondary outcomes were emergency department visits that did not result in hospital admission. Outcome assessment occurred at the patient level. We obtained data for readmissions and emergency department visits from 6 hospitals in central Illinois where study patients were likely to seek care. We validated readmissions and emergency department visits via patient/proxy telephone interviews that occurred 6 months after index hospital discharge. Interviewers were blind to intervention assignment. We evaluated the adequacy of the blind and asked interviewers to guess the patient's intervention assignment.

Another secondary outcome was the proportion of patients who experienced an adverse event related to medical management within 1 month after discharge. For adverse event ascertainment, we employed the process of Forster et al. ${ }^{1,2}$ Within 20 to 40 days after discharge, an internal medicine physician performed telephone interviews with the patient or proxy. The interviewer recorded symptoms, drug information, other treatment, hospital readmissions, and emergency department visits. Another physician compiled case summaries from interview data and information abstracted from the electronic medical record, including dictated discharge summaries from the index hospitalization and postdischarge emergency department visits, diagnostic test results, and readmission reports. Two additional internal medicine physicians adjudicated each case summary separately. We counted adverse events only when adjudicators agreed that medical management probably or definitely caused the event. The initial rating by each adjudi- cator revealed moderate-to-good agreement (Kappa = 0.52). ${ }^{30}$ When initial adjudications were discordant, then adjudicators met and resolved all discrepancies. The adjudicators also scored the severity of the adverse event. The severity scale options were serious laboratory abnormality only, 1 day of symptoms, several days of symptoms, nonpermanent disability, permanent disability, or death. The adjudicators also scored the adverse event as preventable (yes/no), ameliorable (yes/no), and recorded system problems associated with preventable and ameliorable adverse events. ${ }^{1}$ For adverse drug events, the adjudicators recorded preventability categories defined by previous investigators. ${ }^{31}$ We designed the adverse event outcome ascertainment as a blinded process. We evaluated the success of the blind and asked adjudicators to guess the patient's intervention assignment.

\section{Sample Size}

The sample size analysis employed several assumptions regarding the proportion of readmitted patients. The estimated readmission rate after usual care was $37 \% .^{24,32-36}$ The minimum clinically relevant difference in readmission rates was $13 \%$, an empirical boundary for quantitative significance. $^{37}$ Estimates for intracluster correlation were not available when we designed the trial. We projected intracluster correlations with low, medium, and high values. The cluster number and size were selected to maintain test significance level, 1-sided alpha, $<0.05$ and power $>80 \%$. The sample size assumed no interim analysis. The initial sample size estimates were 11 physician clusters per intervention with 25 patients per cluster. During the first 2 months of patient recruitment, we observed that we could not consistently achieve clusters with 25 patients. We recalculated the sample size. Using the same assumptions, we found we could achieve similar test significance and power with 35 physician clusters per intervention and 9 patients per cluster. The sample size calculator was nQuery (Statistical Solutions, Saugus, MA).

\section{Statistical Methods}

Analyses were performed with SPSS PC (Version 15.0.1; SPSS Inc, Chicago, IL). Using descriptive statistics, we reported baseline variables as means and standard deviations (SD) for interval variables, and percentages for categorical variables. For outcome variables, we utilized the principle of intention-to-treat and assumed patient exposure to the intervention randomly assigned to their discharging physician. We inspected scatter plots and correlations for all variables to test assumptions regarding normal distribution, homogeneity of variance, and linearity of relationships between independent and dependent variables. When assumptions failed, we stratified variables (median or thirds) or performed transformations to satisfy assumptions. For patient-level outcome variables, we calculated intracluster correlation coefficients. The assessment of the blind was unaffected by the cluster assumption so we used the chi-

2009 Society of Hospital Medicine DOI 10.1002/jhm.469 Published online in wiley InterScience (www.interscience.wiley.com) 
TABLE 1. Baseline Characteristics for Each Intervention at the Hospital Physician Cluster Level and Individual Patient Level

\begin{tabular}{|c|c|c|}
\hline & $\begin{array}{l}\text { Discharge } \\
\text { Software }\end{array}$ & Usual Care \\
\hline Hospital physician characteristics, n (\%) & $(\mathrm{n}=35)$ & $(\mathrm{n}=35)$ \\
\hline Postgraduate year 1 & $18(51.4)$ & $23(65.7)$ \\
\hline Postgraduate years 2-4 & $10(28.6)$ & $7(20.0)$ \\
\hline Attending physician & $7(20.0)$ & $5(14.3)$ \\
\hline Patient characteristics & $(\mathrm{n}=316)$ & $(\mathrm{n}=315)$ \\
\hline Gender, female, n (\%) & $180(57.0)$ & $168(53.3)$ \\
\hline \multicolumn{3}{|l|}{ Age, years, n (\%) } \\
\hline $18-44$ & $68(21.5)$ & $95(30.2)$ \\
\hline $45-54$ & $79(25.0)$ & $76(24.1)$ \\
\hline $55-64$ & $86(27.2)$ & $74(23.5)$ \\
\hline $65-98$ & $83(26.3)$ & $70(22.2)$ \\
\hline \multicolumn{3}{|l|}{ Race, n (\%) } \\
\hline Caucasian & $239(75.6)$ & $229(72.7)$ \\
\hline Black & $72(22.8)$ & $85(27.0)$ \\
\hline Other & $5(1.6)$ & $1(0.3)$ \\
\hline \multicolumn{3}{|l|}{ Self-rated health status, $\mathrm{n}(\%)$} \\
\hline Poor & $82(25.9)$ & $108(34.3)$ \\
\hline Fair & $169(53.5)$ & $147(46.7)$ \\
\hline Good & $54(17.1)$ & $46(14.6)$ \\
\hline Very good & $10(3.2)$ & $11(3.5)$ \\
\hline Excellent & $1(0.3)$ & $3(1.0)$ \\
\hline Diabetes mellitus, $n(\%)$ & $172(54.4)$ & $177(56.2)$ \\
\hline \multicolumn{3}{|c|}{ Chronic obstructive pulmonary disease, $\mathrm{n}(\%)$} \\
\hline None & $259(82.0)$ & $257(81.6)$ \\
\hline Without oral steroid or home oxygen & $28(8.9)$ & $26(8.3)$ \\
\hline With chronic oral steroid & $10(3.2)$ & $8(2.5)$ \\
\hline With home oxygen \pm oral steroid & $19(6.0)$ & $24(7.6)$ \\
\hline Coronary heart disease, $\mathrm{n}(\%)$ & $133(42.1)$ & $120(38.1)$ \\
\hline Heart failure, n (\%) & $80(25.3)$ & 67 (21.3) \\
\hline Informal caregiver available, yes, $\mathrm{n}(\%)$ & $313(99.1)$ & $313(99.4)$ \\
\hline Taking loop diuretic, n (\%) & $110(34.8)$ & $88(27.9)$ \\
\hline \multicolumn{3}{|l|}{ Physical functioning from SF-36, n (\%) } \\
\hline Lowest third & $128(40.5)$ & $121(38.4)$ \\
\hline Upper two-thirds & $188(59.5)$ & $194(61.6)$ \\
\hline \multicolumn{3}{|l|}{ Mental health from SF-36, n (\%) } \\
\hline Lowest third & $113(35.8)$ & $117(37.1)^{*}$ \\
\hline Upper two-thirds & $203(64.2)$ & $197(62.5)^{*}$ \\
\hline \multicolumn{3}{|l|}{$\begin{array}{l}\text { Hospital admissions during year } \\
\text { prior to index admission, } n(\%)\end{array}$} \\
\hline 0 or 1 & $247(78.2)$ & $224(71.1)$ \\
\hline 2 or more & $69(21.8)$ & $91(28.9)$ \\
\hline \multicolumn{3}{|l|}{ Emergency department visits during } \\
\hline \multicolumn{3}{|l|}{6 months before index admission, $\mathrm{n}(\%)$} \\
\hline 0 or 1 & $194(61.4)$ & $168(53.3)$ \\
\hline 2 or more & $122(38.6)$ & $147(46.7)$ \\
\hline \multicolumn{3}{|l|}{$\begin{array}{l}\text { Outpatient doctor or clinic visits during } \\
\text { year prior to index admission }\end{array}$} \\
\hline 0 to 4 & $97(30.7 \%)$ & $77(24.4 \%)$ \\
\hline 5 to 8 & $68(21.5 \%)$ & $81(25.7 \%)$ \\
\hline 9 to 12 & $82(25.9 \%)$ & $84(26.7 \%)$ \\
\hline 13 or more & $69(21.8 \%)$ & $73(23.2 \%)$ \\
\hline \multicolumn{3}{|l|}{ Insurance or payor } \\
\hline Medicare, age less than 65 years & $18(5.7 \%)$ & $13(4.1 \%)$ \\
\hline Medicare, age 65 years and older & $56(17.7 \%)$ & $40(12.7 \%)$ \\
\hline Medicaid, age less than 65 years & $98(31.0 \%)$ & $130(41.3 \%)$ \\
\hline Medicaid, age 65 years and older & $17(5.4 \%)$ & $20(6.3 \%)$ \\
\hline Commercial or veteran & $85(26.9 \%)$ & $61(19.4 \%)$ \\
\hline Self-pay & $42(13.3 \%)$ & $51(16.2 \%)$ \\
\hline
\end{tabular}

\begin{tabular}{lcc} 
TABLE 1. (Continued) & & \\
& $\begin{array}{l}\text { Discharge } \\
\text { Software }\end{array}$ & Usual Care \\
\hline & & \\
& & \\
Religious participation & $159(50.3 \%)$ & $164(52.1 \%)$ \\
$\quad$ Never & $55(17.4 \%)$ & $51(16.2 \%)$ \\
1-24 times per year & $102(32.3 \%)$ & $100(31.7 \%)$ \\
1-7 times per week & $31(9.8 \%)$ & $39(12.4 \%)$ \\
Volunteer activity, 1 or more hour/month & & \\
Employment status & $229(72.5 \%)$ & $233(74.4 \%)^{*}$ \\
Not working & $30(9.5 \%)$ & $25(8.0 \%)^{*}$ \\
Part-time (<37.5 hours/week) & $57(18.0 \%)$ & $55(17.6 \%)^{*}$ \\
Full-time (at least 37.5 hour/week) & $10.5(4.8)$ & $9.9(5.1)$ \\
Number of discharge medications, mean (SD) & $1.8(1.2)$ & $1.6(1.3)$ \\
Severity of illness, mean (SD) & $1.7(1.4)$ & $1.6(1.9)$ \\
Charlson-Deyo comorbidity, mean (SD) & $3.9(3.5)$ & $3.5(3.5)$ \\
Index hospital length of stay, days, mean (SD) & $17.9(12.9)$ & $19.1(12.9)$ \\
Blood urea nitrogen, mean (SD) & & \\
Probability of repeat admission, Pra, mean (SD) & $0.486(0.072)$ & $0.495(0.076)$ \\
\hline
\end{tabular}

Abbreviation: SD, standard deviation.

* Missing data for 1 or 2 subjects.

square procedure. For analysis of time to event, we used Kaplan-Meier plots.

The primary hypothesis was a significant decrease in the primary readmission outcome for patients assigned to discharge software. We tested the primary hypothesis with generalized estimating equations that corrected for clustering by hospital physician and adjusted for covariates that predicted readmission. The intervention variable was discharge software versus usual care handwritten discharge. We reported parameter estimates of the intervention variable coefficient and Wald $95 \%$ confidence interval $(95 \% \mathrm{CI})$ with and without correction for cluster. For the secondary, patient-level outcomes, we performed similar analyses with generalized estimating equations that corrected for clustering by hospital physician.

During covariate analysis, we screened all baseline variables for their correlation with readmission. The variable with the highest correlation and $P$ value $<0.05$ entered initially in the general estimating equation. After initial variable entry, we evaluated subsequent variables with partial correlations that controlled for variables entered previously. At each iterative step, we entered into the model the variable with the highest partial correlation and $P$ value $<0.05$.

In exploratory analyses, we examined intervention group differences within strata defined by covariates that predicted readmission. We used generalized estimating equations and adjusted for the other covariates that predicted readmission.

\section{Results}

We screened 127 physicians who were general internal medicine hospital physicians. Seventy physicians consented and received random allocation to discharge software or usual 


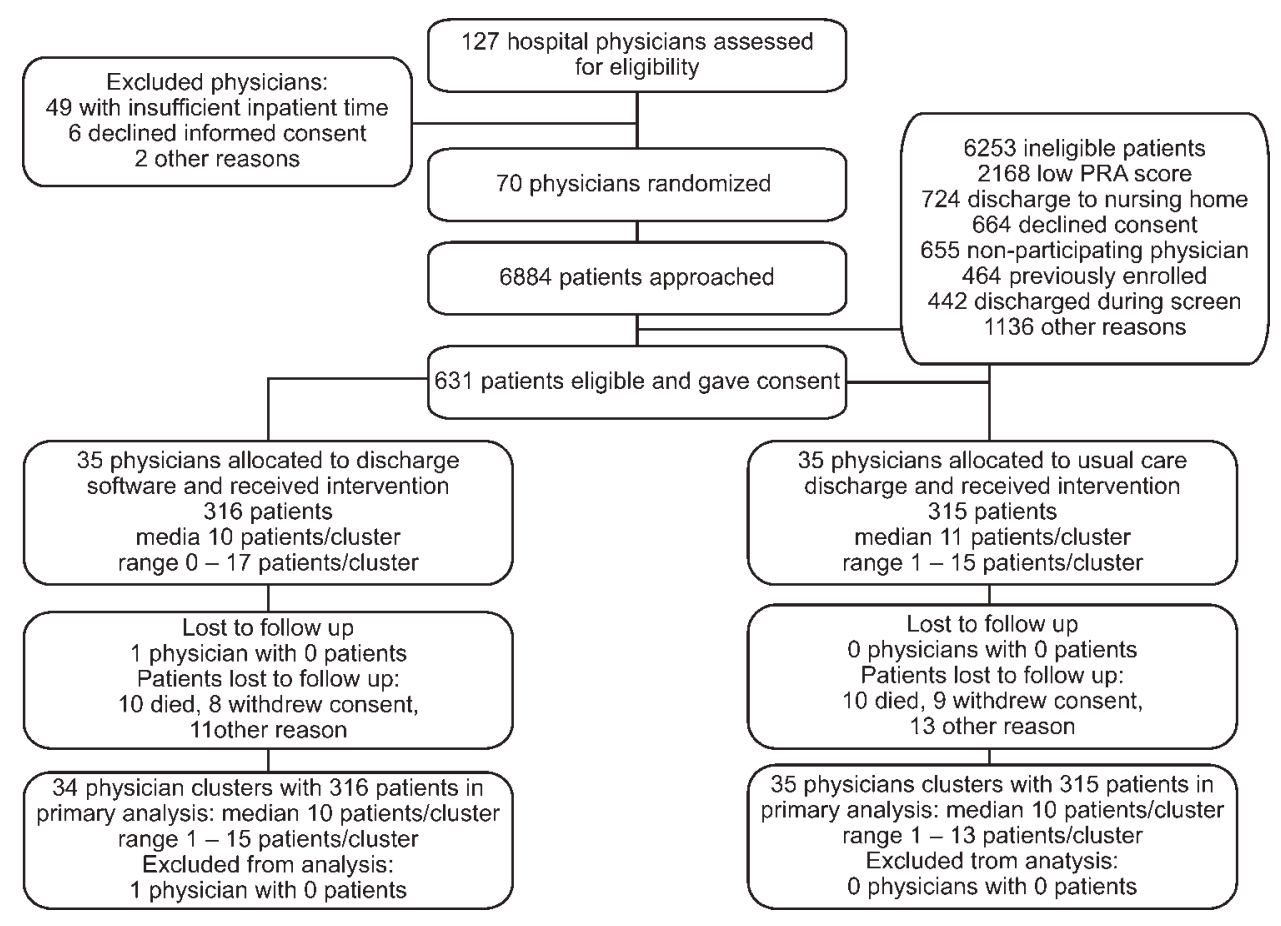

FIGURE 1. Trial flow diagram for hospital physicians and patients.

care. The physician characteristics appear in Table 1. Most of the hospital physicians were interns in the first year of postgraduate training $(58.6 \% ; 41 / 70)$. We excluded 57 physicians for reasons shown in the trial flow diagram (Figure 1). The most common reason for hospital physician exclusion applied to resident physicians in their last months of training before graduation or emergency department residents temporarily assigned to internal medicine training. We approached 6,884 patients during their index hospitalization. After excluding 6,253 ineligible patients, we enrolled and followed 631 patients who received the discharge intervention (Figure 1). During 6 months of follow-up, a small proportion of patients died $(3 \% ; 20 / 631)$. Hospital records were available for deceased patients and they were included in the analysis. A small proportion $(6 \% ; 41 / 631)$ of patients withdrew consent or left the trial for other reasons during 6 months. There was no differential dropout between the interventions. Protocol deviations were rare $(0.5 \% ; 3 / 631)$. Three patients erroneously received usual care discharge from physicians assigned to discharge software. All 631 patients were included in the intention-to-treat analysis. The baseline characteristics of the randomly-assigned hospital physicians and their patients are in Table 1.

We asked outpatient physicians about their receipt of discharge communication from hospital physicians. The text of the question was, "How soon after discharge did you receive any information (in any form) relating to this patient's hospital admission and discharge plans?" We mailed the question 10 days after discharge to outpatient physicians desig- nated by patients enrolled in the study. Among patients in the discharge software group, $75.0 \%$ (237/316) of their outpatient physicians responded to the question. The response rate was $80.6 \%$ (254/315) from physicians who followed patients in the usual care group. Respondents from the discharge software group said "within 1-2 days" or "within a week" for $56.0 \%(177 / 316)$ of patients. Respondents from the usual care group said "within 1-2 days" or "within a week" for $57.1 \%(180 / 315)$ of patients. The difference between the intervention groups, $-1.1 \%(95 \% \mathrm{CI},-9.2 \%$ to $6.9 \%$ ), was not significant.

The primary, prespecified, outcome of the study was the proportion of patients with at least 1 readmission to the hospital. After intervention with discharge software versus usual care, there was no significant difference in readmission rates (Table 2) or time to first readmission (Figure 2). We screened all baseline variables in Table 1 and sought predictors of readmission to employ in adjusted models. For example, we evaluated physician level of training because we wondered if experience or seniority affected readmission when hospital physicians used the discharge software or usual care discharge. The candidate variable, physician level of training, did not correlate with readmission (rho $=$ $-0.066 ; P=0.100$ ), so it was dropped from subsequent analyses. After screening all variables in Table 1 , we found 4 independent predictors of readmission: previous hospitalizations, previous emergency department visits, heart failure, and physical function. Generalized estimating equations for readmission that adjusted for predictor variables confirmed

2009 Society of Hospital Medicine DOI 10.1002/jhm.469 Published online in wiley InterScience (www.interscience.wiley.com). 


\begin{tabular}{|c|c|c|c|c|c|c|}
\hline Outcome & $\begin{array}{l}\text { Discharge } \\
\text { Software, } \\
\text { n (\%) }\end{array}$ & $\begin{array}{l}\text { Usual } \\
\text { Care, } \\
\text { n (\%) }\end{array}$ & $\begin{array}{l}\text { Parameter Estimate } \\
\text { Without Cluster } \\
\text { Correction Intervention } \\
\text { Coefficient }(95 \% \mathrm{CI})\end{array}$ & $\begin{array}{l}P \\
\text { Value }\end{array}$ & $\begin{array}{l}\text { Parameter Estimate } \\
\text { With Cluster Correction } \\
\text { Intervention Coefficient } \\
(95 \% \mathrm{CI})\end{array}$ & $\begin{array}{l}P \\
\text { Value }\end{array}$ \\
\hline \multicolumn{7}{|c|}{$\begin{array}{l}\text { NOTE: Parameter estimates are intervention coefficients from generalized estimating equations for outcome variables. Parameter estimates from ger } \\
\text { clustering by hospital physician: } 34 \text { physicians assigned to discharge software and } 35 \text { assigned to usual care. } \\
\text { Abbreviation: } 95 \% \text { CI, Wald } 95 \% \text { confidence interval. } \\
\text { *Generalized estimating equations adjusted for previous hospitalizations, previous emergency department visits, heart failure, and physical function. }\end{array}$} \\
\hline
\end{tabular}

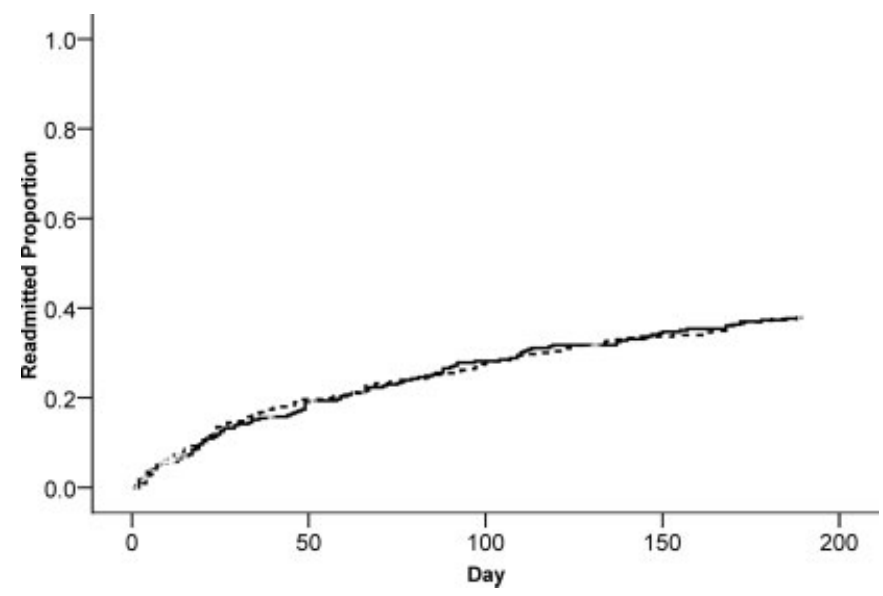

FIGURE 2. Kaplan-Meier curves for first readmission after index hospital discharge for patients assigned to receive discharge software versus usual care discharge. Solid line represents patients assigned to discharge software. Dotted line represents patients assigned to usual care discharge.

a negligible parameter estimate for the discharge intervention variable coefficient (Table 2).

We evaluated emergency department visits that were unrelated to readmission as secondary, prespecified, outcomes. The results were similar to readmission results. While the proportion of patients with at least 1 emergency department visit was lower for the discharge software intervention, the difference with usual care was not significant (Table 2). There was no significant difference between interventions for time to first emergency department visit (Figure 3).

Postdischarge adverse events were secondary, prespecified, outcomes. Data for adverse event adjudication were available for $98 \%(309 / 316)$ of discharge software patients and $97 \%(307 / 315)$ of usual care patients. Within 1 month after discharge, 46 patients had adverse events probably or definitely related to medical management. Two patients had

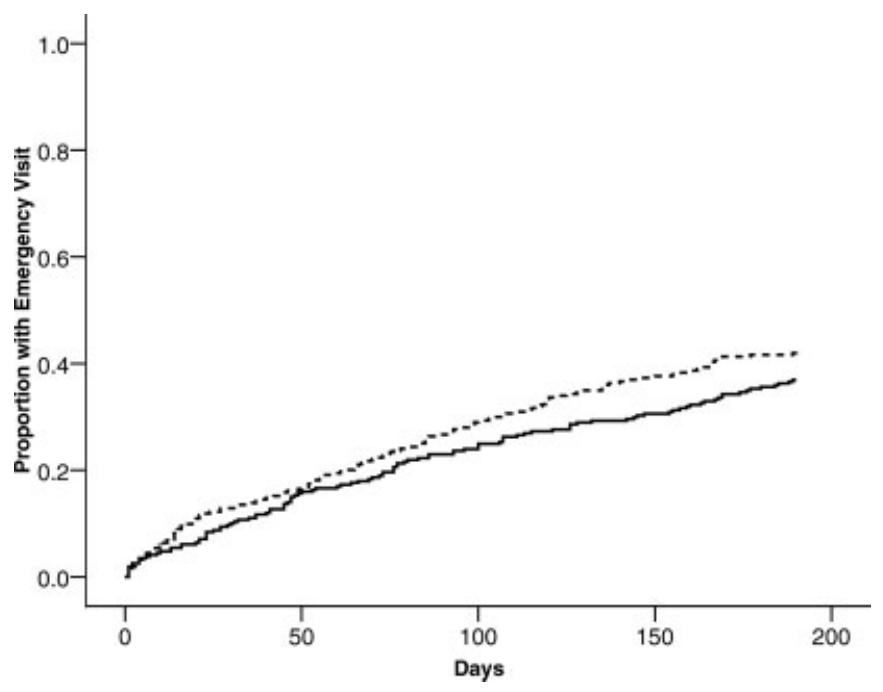

FIGURE 3. Kaplan-Meier curves for first emergency department visit after index hospital discharge for patients assigned to receive discharge software versus usual care discharge. Solid line represents patients assigned to discharge software. Dotted line represents patients assigned to usual care discharge.

2 events and 1 patient had 3 events. For analysis, we randomly selected 1 event per patient. When comparing patients assigned to discharge software versus usual care, there were no differences in adverse events related to medical management (Table 2). Most of the events were possible adverse drug events $(74 \% ; 34 / 46)$. The adverse event severity was several days of symptoms or nonpermanent disability for $76 \%(35 / 46)$ of the adverse events. Adjudicators rated $26 \%(12 / 46)$ of the adverse events as preventable and $46 \%$ $(21 / 46)$ as ameliorable. The absolute numbers of events were small. There were no differences between discharge software and usual care patients within adverse event strata defined by type, severity, preventable, or ameliorable (Table 3). For most of the patients with adverse events, the 


\begin{tabular}{|c|c|c|}
\hline & $\begin{array}{l}\text { Discharge } \\
\text { Software (n) }\end{array}$ & $\begin{array}{l}\text { Usual Care } \\
\text { Discharge (n) }\end{array}$ \\
\hline At least 1 adverse event & 23 & 23 \\
\hline Preventable adverse event & 7 & 5 \\
\hline Ameliorable adverse event & 9 & 12 \\
\hline \multicolumn{3}{|l|}{ Adverse event severity } \\
\hline Serious laboratory abnormality only or 1 day of symptoms & 5 & 5 \\
\hline Several days of symptoms or nonpermanent disability & 18 & 17 \\
\hline Permanent disability or death & 0 & 1 \\
\hline \multicolumn{3}{|l|}{ Adverse event by type } \\
\hline Possible adverse drug event & 17 & 17 \\
\hline Procedure-related injury & 2 & 1 \\
\hline Therapeutic error & 4 & 4 \\
\hline Diagnostic error & 0 & 1 \\
\hline \multicolumn{3}{|l|}{ System problems associated with preventable or ameliorable adverse events } \\
\hline Inadequate patient education regarding the medical condition or its treatment & 0 & 1 \\
\hline Poor communication between patient and physician & 2 & 1 \\
\hline Poor communication between hospital and community physicians & 0 & 0 \\
\hline Inadequate monitoring of the patient's illness after discharge & 0 & 6 \\
\hline Inadequate monitoring of the patient's treatment after discharge & 2 & 6 \\
\hline No emergency contact number given to patient to call about problems & 0 & 0 \\
\hline Patient with problems getting prescribed medications immediately & 1 & 0 \\
\hline Inadequate home services & 0 & 0 \\
\hline Delayed follow-up care & 0 & 3 \\
\hline Premature hospital discharge & 1 & 2 \\
\hline \multicolumn{3}{|l|}{ Adverse drug event (ADE) preventability categories } \\
\hline Drug involved in the ADE inappropriate for the clinical condition & 2 & 4 \\
\hline Dose, route, or frequency inappropriate for age, weight, creatinine clearance, or disease & 1 & 2 \\
\hline Failure to obtain required lab tests and/or drug levels & 1 & 2 \\
\hline Prior history of an adverse event or allergy to the drug & 1 & 2 \\
\hline Drug-drug interaction involved in the $\mathrm{ADE}$ & 2 & 0 \\
\hline Toxic serum drug level documented & 0 & 0 \\
\hline Noncompliance involved in the $\mathrm{ADE}$ & 0 & 1 \\
\hline
\end{tabular}

adjudicators could not identify a system problem or preventability category (Table 3). When a deficiency was evident, there was no pattern to suggest a significant difference between discharge software patients versus usual care patients.

When we designed the trial, we assumed variance in outcomes measured at the patient level. We predicted some variance attributable to clustering by hospital physician. After the trial, we calculated the intracluster correlation coefficients for readmissions, emergency department visits, and adverse events. For all of these outcomes, the intracluster correlation coefficients were negligible. We also evaluated generalized estimating equations with and without correction for hospital physician cluster. We confirmed the negligible cluster effect on confidence intervals for intervention coefficients (Table 2).

We performed an exploratory stratified analysis. We evaluated the intervention effect on readmission within subgroups defined by covariates that predicted readmission (Table 4). When the intervention groups were compared within baseline categories of previous hospitalizations, previous emergency department visits, heart failure, and physical functioning, there was a consistent pattern with no differential effect by intervention assignment. None of the intervention coefficients were statistically significant (Table 4).

\section{Assessment of the Success of the Blind}

We evaluated the adequacy of the blind for outcome assessors who interviewed patients or adjudicated adverse events. The guesses of outcomes assessors were unrelated to true intervention assignment (all $P$ values $>0.097$ ). We interpreted the blind as adequate for outcome assessors who recorded readmissions, emergency department visits, and adverse events.

\section{Discussion}

We performed a cluster-randomized clinical trial to measure the effects of discharge software versus usual care handwritten discharge. The discharge software with CPOE 
TABLE 4. Patients Readmitted At Least Once Within 6 Months by Subgroup

\begin{tabular}{|c|c|c|c|}
\hline Subgroup & $\begin{array}{l}\text { Discharge Software } \\
\text { Readmitted } n / n(\%)\end{array}$ & $\begin{array}{l}\text { Usual Care } \\
\text { Readmitted n/n (\%) }\end{array}$ & $\begin{array}{l}\text { Adjusted Parameter } \\
\text { Estimate Intervention } \\
\text { Coefficient }(95 \% \mathrm{CI})\end{array}$ \\
\hline \multicolumn{4}{|c|}{ Hospital admissions during year prior to index admission } \\
\hline 0 or 1 & $77 / 247(31.2)$ & $73 / 224(32.6)$ & $-0.025(-0.095,0.045)^{*}$ \\
\hline 2 or more & $40 / 69(58.0)$ & $46 / 91(50.5)$ & $0.059(-0.090,0.208)^{*}$ \\
\hline \multicolumn{4}{|c|}{ Emergency department visits during 6 months before index admission } \\
\hline \multicolumn{4}{|l|}{ Heart failure } \\
\hline Present & $40 / 80(50.0)$ & $36 / 67(53.7)$ & $-0.024(-0.224,0.177)^{\ddagger}$ \\
\hline Absent & $77 / 236(32.6)$ & $83 / 248(33.5)$ & $0.000(-0.076,0.075)^{\ddagger}$ \\
\hline \multicolumn{4}{|c|}{ Physical functioning from SF-36 } \\
\hline Lowest third & $55 / 128(43.0)$ & $59 / 121(48.8)$ & $-0.032(-0.161,0.096)^{\S}$ \\
\hline Upper two-thirds & $62 / 188(33.0)$ & $60 / 194(30.9)$ & $0.012(-0.071,0.095)^{\S}$ \\
\hline \multicolumn{4}{|c|}{ Abbreviation: $95 \%$ CI, Wald $95 \%$ confidence interval. } \\
\hline \multicolumn{4}{|c|}{ *Adjusted for previous emergency department visits, heart failure, and physical functioning. } \\
\hline \multicolumn{4}{|c|}{${ }^{\dagger}$ Adjusted for previous hospital admissions, heart failure, and physical functioning. } \\
\hline \multicolumn{4}{|c|}{${ }^{\ddagger}$ Adjusted for previous hospital admissions, previous emergency department visits, and physical functioning. } \\
\hline${ }^{\$}$ Adjusted for previous $\mathrm{h}$ & & & \\
\hline
\end{tabular}

implemented elements of high-quality discharge planning and communication endorsed by the National Quality Forum and systematic reviews. ${ }^{6,38}$ Despite theoretical benefits, our discharge software intervention did not reduce readmissions or emergency department visits. What were potential explanations for our results? We assumed an association between postdischarge adverse events and readmissions or emergency department visits. ${ }^{1}$ Our failure to reduce adverse events might explain the failure to reduce readmissions or emergency department visits. Another potential explanation was related to adverse drug events. Other investigators showed most postdischarge adverse events were adverse drug events and our data confirmed previous studies. ${ }^{1,2}$ Medication reconciliation at discharge was a potential mechanism for adverse drug event reduction. ${ }^{14}$ Medication reconciliation was the standard at the study hospital, so it was unethical to deny reconciliation to patients assigned to either intervention. ${ }^{39}$ Required medication reconciliation in both groups, by its known effect on preventable adverse drug events, might have reduced the event rates in both groups. ${ }^{14}$ This possibility is supported by the low rate of adverse events observed in our study compared with other studies. ${ }^{1}$ We speculate that the low background rate of adverse events at the study hospital may have minimized events in both the discharge software and usual care groups and prevented detection of software benefits, if present. $^{39}$

One limitation of our study may have been the discharge software. The automated decision support in our software lacked features that might have improved outcomes. For example, the software did not generate a list of diagnostic test results that were pending at the time of discharge. Our software relied on prompts to the physician user that did not specify which tests were pending. The software did not perform error checks on the discharge orders to warn physicians about drug-drug interactions, therapeutic duplications, or missing items (eg, immunizations, drugs, education). The absence of these software enhancements made our discharge process vulnerable to the lapses and slips of the physician user. Whether or not such enhancements affect clinically relevant outcomes remains a testable hypothesis for future studies.

Another limitation of our study was the outpatient physician response. Discharge software did not increase the proportion of outpatient physicians who said they received communication within 7 days after hospital discharge. Our intervention addressed the sending partner but not the receiving partner in the communication dyad. Our discharge software was not designed to change information flow within the outpatient physician office. We do not know if discharge communication arrived and remained unnoticed until the patient called or visited the outpatient clinic. Future studies of discharge communication should consider a closed loop design to assure receipt and comprehension.

When we designed our study, we expected at least some variance between patient clusters attributable to the physician who performed the discharge. Our analysis of intracluster correlation revealed negligible variance. We speculate the highly-standardized discharge process implemented by discharge software and usual care at our hospital resulted in minimal variance. Future studies of discharge interventions may consider designs that avoid cluster randomization. 
In conclusion, a discharge software application of $\mathrm{CPOE}$ did not affect readmissions, emergency department visits, or adverse events after discharge.

\section{Acknowledgements}

The authors thank Howard S. Cohen, MD, for his review of the trial protocol and the manuscript.

\section{Address for correspondence and reprint requests:}

James F. Graumlich, MD, Department of Medicine, 530 NE Glen Oak Avenue, Peoria, IL 61637; Telephone: 309-655-7734; Fax: 309-6557732; E-mail: jfg@uic.edu Received 18 January 2008; revision received 9 November 2008; accepted 16 December 2008.

\section{References}

1. Forster AJ, Murff HJ, Peterson JF, Gandhi TK, Bates DW. The incidence and severity of adverse events affecting patients after discharge from the hospital. Ann Intern Med. 2003;138(3):161-167.

2. Forster AJ, Clark HD, Menard A, et al. Adverse events among medical patients after discharge from hospital. CMAJ. 2004;170(3):345-349.

3. Epstein K, Juarez E, Loya K, Gorman MJ, Singer A. Frequency of new or worsening symptoms in the posthospitalization period. J Hosp Med. 2007;2(2):58-68.

4. Johnson A, Sandford J, Tyndall J. Written and verbal information versus verbal information only for patients being discharged from acute hospital settings to home. Cochrane Database Syst Rev. 2003;(4):CD003716.

5. Shepperd S, Parkes J, McClaren J, Phillips C. Discharge planning from hospital to home. Cochrane Database Syst Rev. 2004;(1):CD000313.

6. Kripalani S, LeFevre F, Phillips CO, Williams MV, Basaviah P, Baker DW. Deficits in communication and information transfer between hospitalbased and primary care physicians: implications for patient safety and continuity of care. JAMA. 2007;297(8):831-841.

7. Nace GS, Graumlich JF, Aldag JC. Software design to facilitate information transfer at hospital discharge. Inform Prim Care. 2006;14(2):109-119.

8. Kaushal R, Shojania KG, Bates DW. Effects of computerized physician order entry and clinical decision support systems on medication safety: a systematic review. Arch Intern Med. 2003;163(12):1409-1416.

9. Kuperman GJ, Gibson RF. Computer physician order entry: benefits, costs, and issues. Ann Intern Med. 2003;139(1): 31-39.

10. Chaudhry B, Wang J, Wu S, et al. Systematic review: impact of health information technology on quality, efficiency, and costs of medical care. Ann Intern Med. 2006;144(10):742-752.

11. Kiefe CI, Heudebert G, Box JB, Farmer RM, Michael M, Clancy CM. Compliance with post-hospitalization follow-up visits: rationing by inconvenience? Ethn Dis. 1999;9(3):387-395.

12. Dexter PR, Perkins S, Overhage JM, Maharry K, Kohler RB, McDonald CJ. A computerized reminder system to increase the use of preventive care for hospitalized patients. N Engl J Med. 2001;345(13):965-970.

13. Paquette-Lamontagne N, McLean WM, Besse L, Cusson J. Evaluation of a new integrated discharge prescription form. Ann Pharmacother. 2001; 35(7-8):953-958.

14. Schnipper JL, Kirwin JL, Cotugno MC, et al. Role of pharmacist counseling in preventing adverse drug events after hospitalization. Arch Intern Med. 2006;166(5):565-571.

15. Marcantonio ER, McKean S, Goldfinger M, Kleefield S, Yurkofsky M, Brennan TA. Factors associated with unplanned hospital readmission among patients 65 years of age and older in a Medicare managed care plan. Am J Med. 1999;107(1):13-17.

16. Sands DZ, Safran C. Closing the loop of patient care-a clinical trial of a computerized discharge medication program. Proc Annu Symp Comput Appl Med Care. 1994:841-845.

17. O'Connell EM, Teich JM, Pedraza LA, Thomas D. A comprehensive inpatient discharge system. Proc AMIA Annu Fall Symp. 1996:699-703.

18. Agency for Healthcare Research and Quality. Making health care safer: a critical analysis of patient safety practices, subchapter 42.3. Discharge summaries and follow-up. Available at: http://www.ahrq.gov/clinic/ptsafety/ chap42b. htm\#42.3. Accessed January 2009.

19. Pacala JT, Boult C, Boult L. Predictive validity of a questionnaire that identifies older persons at risk for hospital admission. J Am Geriatr Soc. 1995;43(4):374-377.

20. Pacala JT, Boult C, Reed RL, Aliberti E. Predictive validity of the Pra instrument among older recipients of managed care. J Am Geriatr Soc. 1997;45(5):614-617.

21. Graumlich JF, Novotny NL, Aldag JC. Brief scale measuring patient preparedness for hospital discharge to home: psychometric properties. J Hosp Med. 2008;3(6):446-454.

22. Ware JE Jr. SF-36 health survey update. Spine. 2000;25(24):3130-3139.

23. Reuben DB, Keeler E, Seeman TE, Sewall A, Hirsch SH, Guralnik JM. Development of a method to identify seniors at high risk for high hospital utilization. Med Care. 2002;40(9):782-793.

24. Naylor MD, Brooten D, Campbell R, et al. Comprehensive discharge planning and home follow-up of hospitalized elders: arandomized clinical trial. JAMA. 1999;281(7):613-620.

25. Smith DM, Giobbie-Hurder A, Weinberger M, et al. Predicting non-elective hospital readmissions: a multi-site study. Department of Veterans Affairs Cooperative Study Group on Primary Care and Readmissions. J Clin Epidemiol. 2000;53:1113-1118.

26. Corrigan JM, Martin JB. Identification of factors associated with hospital readmission and development of a predictive model. Health Serv Res. 1992;27(1):81-101.

27. Romano PS, Chan BK. Risk-adjusting acute myocardial infarction mortality: are APR-DRGs the right tool? Health Serv Res. 2000;34(7):1469-1489.

28. Deyo RA, Cherkin DC, Ciol MA. Adapting a clinical comorbidity index for use with ICD-9-CM administrative databases. J Clin Epidemiol. 1992; 45(6):613-619.

29. Shelton P, Sager MA, Schraeder C. The community assessment risk screen (CARS): identifying elderly persons at risk for hospitalization or emergency department visit. Am J Manag Care. 2000;6(8):925-933.

30. Sackett DL, Haynes RB, Guyatt GH, Tugwell P. Clinical Epidemiology: A Basic Science for Clinical Medicine. 2nd ed. Boston: Little, Brown; 1991.

31. Winterstein AG, Hatton RC, Gonzalez-Rothi R, Johns TE, Segal R. Identifying clinically significant preventable adverse drug events through a hospital's database of adverse drug reaction reports. Am J Health Syst Pharm. 2002;59(18):1742-1749.

32. Nazareth I, Burton A, Shulman S, Smith P, Haines A, Timberal H. A pharmacy discharge plan for hospitalized elderly patients-a randomized controlled trial. Age Ageing. 2001;30(1):33-40.

33. McInnes E, Mira M, Atkin N, Kennedy P, Cullen J. Can GP input into discharge planning result in better outcomes for the frail aged: results from a randomized controlled trial. Fam Pract. 1999;16(3):289-293.

34. Phillips CO, Wright SM, Kern DE, Singa RM, Shepperd S, Rubin HR. Comprehensive discharge planning with postdischarge support for older patients with congestive heart failure: a meta-analysis. JAMA. 2004; 291(11):1358-1367.

35. Andersen HE, Schultz-Larsen K, Kreiner S, Forchhammer BH, Eriksen K, Brown A. Can readmission after stroke be prevented? Results of a randomized clinical study: a postdischarge follow-up service for stroke survivors. Stroke. 2000;31(5):1038-1045.

36. Weinberger M, Oddone EZ, Henderson WG. Does increased access to primary care reduce hospital readmissions? Veterans Affairs Cooperative Study Group on Primary Care and Hospital Readmission. $N$ Engl J Med. 1996;334(22):1441-147.

37. Burnand B, Kernan WN, Feinstein AR. Indexes and boundaries for "quantitative significance" in statistical decisions. J Clin Epidemiol. 1990; 43(12):1273-1284.

38. National Quality Forum. Safe Practices for Better Healthcare 2006 Update, A Consensus Report, Safe Practice 11: Discharge Systems. Available at: http://qualityforum.org/pdf/reports/safe_practices/txsppublic.pdf. Accessed January 2009.

39. Whittington J, Cohen H. OSF Healthcare's journey in patient safety. Qual Manag Health Care. 2004;13(1):53-59. 